\title{
Higher Complication Rate Following Hip Fractures in Uremic Patients Undergoing Maintenance Hemodialysis: A Cohort Study
}

\author{
Yi-Hsun Yu*, Yung-Heng Hsu, Ying-Chao Chou, I-Chuan Tseng, Wen-Lin Yeh, Chi-Chung Wu \\ Division of Orthopedic Traumatology, Department of Orthopedic Surgery, Musculoskeletal Research Center, Chang Gung \\ Memorial Hospital, Taiwan \\ Email: *alanyu1007@gmail.com
}

How to cite this paper: Yu, Y.-H., Hsu, Y.-H., Chou, Y.-C., Tseng, I.-C., Yeh, W.-L. and Wu, C.-C. (2018) Higher Complication Rate Following Hip Fractures in Uremic Patients Undergoing Maintenance Hemodialysis: A Cohort Study. Open Access Library Journal, 5: e3986.

https://doi.org/10.4236/oalib.1103986

Received: September 28, 2017

Accepted: February 20, 2018

Published: February 23, 2018

Copyright $\odot 2018$ by authors and Open Access Library Inc.

This work is licensed under the Creative Commons Attribution International License (CC BY 4.0).

http://creativecommons.org/licenses/by/4.0/

\begin{abstract}
The current study retrospectively reviewed the surgical outcomes following hip fractures in uremic patients undergoing long-term hemodialysis and compared the results with those of geriatric patients with osteoporotic hip fractures during 1-year period. Thirteen complications occurred among the 21 uremic patients who underwent surgical intervention for a hip fracture. Two complications were related to the failure of osteosynthesis procedures after femoral neck fractures. While comparing perioperative parameters between the 2 groups, patients in the uremic group had younger age, more comorbidities, lower preoperative hemoglobin levels and platelet counts, and an increased requirement of perioperative blood transfusion. Statistical analysis revealed a significant increase in incidence of postoperative complications in the uremic patients; however, the incidence of surgery-related complications was unchanged. In conclusion, the present study shows that the incidence of perioperative complications in hip fracture surgeries is higher in uremic patients than those in geriatric hip fractures, mainly resulting from their comorbidities. In addition, we recommend hip prosthesis replacement surgery for femoral neck fractures in uremic patients due to the high rate of implant failure.
\end{abstract}

\section{Subject Areas}

Hematology, Surgery \& Surgical Specialties

\section{Keywords}

End-Stage Renal Disease, Uremia, Hip Fracture, Osteosynthesis Surgery, Postoperative Complication 


\section{Introduction}

With the advances in hemodialysis treatment, patients with end-stage renal disease (ESRD) now have a longer survival time and a better quality of life. The mineral and endocrine disturbances that occur with long-term hemodialysis alter skeletal metabolism and contribute to fractures [1] [2] [3]. Additionally, the down-regulation of osteoblast activity as a result of renal osteodystrophy impairs fracture healing in patients with ESRD [4]. The majority of orthopedic fractures in uremic patients are lower limb fractures, especially hip fractures [5] [6] [7] [8]. Hip fractures in uremic patients are associated with high morbidity and mortality, with rates 17 - 20 times higher than those of patients with hip fracture in the general population [1] [2] [9]. The rate of postoperative complications following surgical repair of hip fractures in the ESRD population is also significantly increased [10] [11].

Another population of patients with increased incidence of hip fracture is the geriatric population. Surgical repair for geriatric osteoporotic hip fractures remains a challenge for orthopedic surgeons. Poor bone quality leads to a fixation dilemma, and comorbidities in the elderly may negatively affect surgical outcomes [1] [12] [13] [14] [15]. A similar predicament exists in uremic patients. In ESRD patients with hip fractures, an increased rate of complications and a higher mortality rate after index surgery have been reported in the literature [9] [10] [11] [16]. However, little evidence exists comparing the complications, morbidity, and mortality following surgical repair of hip fractures between uremic and geriatric patients.

The purpose of the present study was to review the surgical outcomes following hip fracture repairs in uremic patients undergoing maintenance hemodialysis. In addition, we compared the results in this specific population with those of non-uremic patients with osteoporotic hip fractures and with a minimum of 12 months postoperative follow-up.

\section{Materials and Methods}

We retrospectively collected patient data between January and December 2013 from the Trauma Registration System in Chang Gung Memorial Hospital, a level one trauma center in northern Taiwan. We only included patients with uremia and hip fractures (femoral neck and femoral intertrochanteric fractures) who underwent maintenance hemodialysis during the study period and before surgery. Four uremic patients with hip fractures were unable to tolerate anesthesia and surgery and were excluded from the data analysis. Patients undergoing peritoneal dialysis for chronic renal failure rather than hemodialysis were also excluded. In addition, uremic patients with poly trauma and multiple fractures were excluded. In total, 21 patients met the inclusion criteria and were studied.

During the same one year review period, we identified 458 consecutive patients from the same database with normal renal function, who underwent surgery for hip fractures. Patients aged less than 60 years were excluded, according 
to the definition of elderly from the World Health Organization. Patients with high-energy trauma, fractures in more than two locations, and poly trauma with more than one major organ injury were also excluded. This was considered necessary as multiple fractures or poly trauma could have confounded the outcome evaluation. Following the exclusion process, 309 geriatric patients with hip fractures were included as the normal control group.

The patient data were recorded prospectively and analyzed retrospectively. The preoperative clinical variables were recorded, including demographic data and laboratory data. The laboratory data included serum white blood cell count, hemoglobin level, platelet count, blood urea nitrogen level, creatinine level, estimated creatinine clearance rate, and prothrombin time. Prothrombin time was documented using the international normalized ratio. The volume of blood loss intraoperatively, the need for blood transfusion during the surgery and perioperative complications were also recorded. Blood transfusions were documented as packed red blood cells (PRBC) and fresh frozen plasma (FFP). The need for transfusion was determined using clinical data, including a postoperative hemoglobin level less than $10 \mathrm{~g} / \mathrm{dL}$ in the control group, a loss of $15 \%$ of total blood volume, and signs of tachycardia or hypotension during or after surgery.

A "complication" in the current study was defined as any additional problems arising after the index surgery beyond the surgeon's expectation, regardless of whether or not the problem was directly related to the surgery. Principal complications related to the hip fracture surgery included the loss of reduction and fixation of the fracture, iatrogenic nerve or vessel injury during the surgery, dislocation of the hip joint after prosthesis replacement, surgical site infection, and loosening of implants. Additional medical or surgical treatments related the patients' comorbidities were also considered complications.

The statistical analysis was conducted using SPSS 18.0 (SPSS Inc., Chicago, IL, USA). Continuous variables were presented as the mean and standard deviation. We compared the preoperative and perioperative variables between the study and control groups using a parametric $t$-test and nonparametric Mann-Whitney U-test. Categorical variables were reported as the frequency, and we compared the two groups using a parametric chi-square test. A two-tailed $P$-value of 0.05 was considered significant.

\section{Results}

Among the 21 uremic patients, one patient died of pneumonia and sepsis during the 12-month follow-up. Overall, there were 13 complications (complication rate: $61.9 \%$ ) during the 12 months following surgery. Eight complications were in patients with femoral neck fracture and five were in patients with femoral intertrochanteric fracture. In patients with femoral neck fracture, two complications were directly related to the failure of osteosynthesis surgery, whereas no surgery-related complications were seen in the patients who underwent prosthe- 
sis replacement surgery. In patients with femoral intertrochanteric fracture, all complications were directly related to preexisting medical comorbidities. Table 1 summarizes the complications and required treatments for patients in the uremic group.

Table 2 and Table 3 show the demographic data, including patient age and sex, as well as perioperative data, including blood test results and American Society of Anesthesiologists (ASA) classification of both the study and control groups. The uremic patients were significantly younger (69.9 \pm 8.5 years) than those in the control group $(78.7 \pm 10.5$ years $)(P<0.001, t$-test). The total number of the underlying comorbidities in the uremic group (3.0 \pm 1.4$)$ was significantly higher than in the control group $(1.4 \pm 1.2)(P<0.001, t$-test $)$.

Although the mean length of time between arrival in emergency department (ED) and surgery in the uremic group $(4 \pm 3.9$ days $)$ was greater than in the control group $(2.7 \pm 2.7$ days), there was no statistical difference $(P=0.136$, Mann-Whitney U-test). Preoperatively, the hemoglobin level was significantly lower in the uremic group $(10.3 \pm 1.3 \mathrm{mg} / \mathrm{dL})$ than in the control group $(11.8 \pm$ $2.0 \mathrm{mg} / \mathrm{dL})(P<0.001, t$-test $)$, as was the platelet count $(152.9 \pm 58.4$ vs. $187.5 \pm$ 72.1, $P=0.032, t$-test).

Table 4 shows postoperative results. The comparison of the number of units of PRBC and FFP transfused revealed a statistical difference $(P<0.001$, Mann-Whitney U-test). Analysis of the variables revealed that there were significantly higher complication rate in the uremic group than in the control group (13/21 vs. 61/309) $(P<0.001$, chi-square test). However, there was no significant difference when comparing the surgery-related complications between the two

Table 1. Complications within 12 months of surgery in the uremic group.

\begin{tabular}{ccccc}
\hline Time of Complication & $\begin{array}{c}\text { Fracture } \\
\text { location }\end{array}$ & Type of Surgery & Complication & Management \\
\hline During admission & FN & Hemiarthroplasty & Acute myocardial infarction & Coronary artery bypass surgery \\
& FN & Hemiarthroplasty & A-V fistula occlusion & Surgical thrombectomy \\
& IT & Osteosynthesis & A-V fistula occlusion & Surgical thrombectomy \\
Within 30 days & IT & Osteosynthesis & A-V fistula occlusion & Surgical thrombectomy \\
& IT & Osteosynthesis & & Surgical thrombectomy \\
& FN & Hemiarthroplasty & Peptic ulcer with bleeding & Endoscopic treatment \\
& IT & Osteosynthesis & Heart failure & Medication control \\
& FN & Osteosynthesis & Hepatic encephalopathy & Medication control \\
& FN & Hemiarthroplasty & Contralateral femoral neck fracture & Bipolar arthroplasty \\
Within 12 months & FN & Osteosynthesis & Implant failure & Bipolar arthroplasty \\
& FN & Osteosynthesis & Implant failure & Total hip arthroplasty \\
& FN & Hemiathroplasty & Pneumonia & Intubation with ventilator support (patient died)
\end{tabular}

FN: Femoral neck; IT: Intertrochanteric area; A-V fistula: arteriovenous fistula. 
Table 2. Demographics of the uremic and control patients.

\begin{tabular}{cccc}
\hline & Uremic group $(\mathrm{n}=21)$ & Control group $(\mathrm{n}=309)$ & $P$-value \\
\hline Age (years) & $69.9 \pm 8.5$ & $78.7 \pm 10.5$ & $<0.001^{*}$ \\
Sex (M: F) & $6: 15$ & $114: 195$ & - \\
Number of comorbidities (n) & $3.0 \pm 1.4$ & $1.4 \pm 1.2$ & $<0.001^{*}$ \\
Cardiovascular disease & 15 & 122 & 0.005 \\
Diabetes mellitus & 13 & 95 & 0.007 \\
Hepatitis & 4 & 15 & 0.025 \\
COPD & 10 & 20 & $<0.001^{*}$ \\
Peptic ulcer & 2 & 29 & 1.000 \\
Cancer & 2 & 25 & 1.000 \\
Cerebrovascular accident & 3 & 59 & 0.776 \\
ESRD & 21 & - & - \\
Fracture location (n) & 11 & 142 & - \\
Intracapsular femoral neck & 10 & 157 & - \\
Intertrochanteric area & & & 0.448 \\
Type of surgery (n) & 13 & & - \\
Osteosynthesis & & 129 & - \\
Prosthesis replacement & & & \\
\hline
\end{tabular}

COPD: chronic obstructive pulmonary disease; ESRD: end-stage renal disease. ${ }^{*}$ Statistical significance, $t$-test.

Table 3. Perioperative factors in the uremic and control patients.

\begin{tabular}{cccc}
\hline & Uremic group $(\mathrm{n}=21)$ & Control group $(\mathrm{n}=309)$ & $P$-value \\
\hline Time from ED to surgery (days) & $4 \pm 3.9$ & $2.7 \pm 2.7$ & 0.136 \\
Blood test & & & \\
White blood cell count (u/mL) & $9857.1 \pm 3894.7$ & $10,514.9 \pm 3731.9$ & 0.436 \\
Hemoglobin (mg/dL) & $10.3 \pm 1.3$ & $11.8 \pm 2.0$ & $<0.001^{*}$ \\
Platelet count & $152.9 \pm 58.4$ & $187.5 \pm 72.1$ & $0.032^{\star}$ \\
INR & $1.1 \pm 0.1$ & $1.1 \pm 1.2$ & 0.95 \\
BUN (mg/dL) & $48.1 \pm 27.8$ & $21.5 \pm 12.9$ & $<0.001^{*}$ \\
Cr (mg/dL) & $5.8 \pm 2.1$ & $1.1 \pm 0.9$ & $<0.001^{\star}$ \\
eCCr & $9.3 \pm 4.5$ & $70.3 \pm 47.3$ & $<0.001^{\star}$ \\
ASA classification & $3.1 \pm 1.2$ & $2.9 \pm 0.3$ & 0.104 \\
\hline
\end{tabular}

ED: emergency department; INR: international normalized ratio; BUN: blood urea nitrogen. Cr: creatinine; eCCr: estimated creatinine clearance rate; ASA: American Society of Anesthesiologists. ${ }^{\star}$ Statistical significance, $t$-test.

groups $(P=0.127$, chi square test $)$.

\section{Discussion}

According to data from the US Renal Data System and the Taiwan National 
Table 4. Postoperative factors in the uremic and control patients.

\begin{tabular}{cccc}
\hline & Uremic group $(\mathrm{n}=21)$ & Control group $(\mathrm{n}=309)$ & $P$-value \\
\hline Total blood loss $(\mathrm{mL})$ & $138.2 \pm 98.3$ & $181.6 \pm 124.8$ & 0.120 \\
Blood transfusion (units) & & & \\
Packed red blood cell & $0.7 \pm 0.9$ & $0.6 \pm 1.0$ & $<0.001^{\dagger}$ \\
Fresh frozen plasma & $0.2 \pm 0.6$ & $0.1 \pm 0.6$ & $<0.001^{\dagger}$ \\
Duration of antibiotics use (days) & $2.1 \pm 1.4$ & $2.5 \pm 2.1$ & 0.316 \\
Admission length (days) & $11.1 \pm 7.6$ & $9.5 \pm 4.6$ & 0.139 \\
Complications (n) & 13 & 61 & $0.001^{\star}$ \\
In hospital & 3 & 32 & 0.389 \\
Within 30 days & 6 & 25 & $0.008^{\star}$ \\
Within 12 months & 4 & 8 & $0.019^{*}$ \\
Surgery-related & 2 & 8 & 0.127 \\
In hospital Mortality & 0 & 8 & 1.000 \\
Re do surgery (n) & 2 & 19 & 0.128 \\
\hline
\end{tabular}

*Statistical significance, $t$-test.

Health Insurance Research Database, Taiwan has the greatest incidence and the second highest prevalence of ESRD in the world since 2000 [17] [18]. Recent advances in hemodialysis have improved the life expectancy and quality of life of ESRD patients. In addition, the advancements in anesthesia, orthopedic implants, and surgical techniques have made elective orthopedic surgery safer in uremic patients [19]. However, for unanticipated surgery such as hip fracture repair, the outcomes remain unpredictable. Hip fractures have been shown to be strongly related to ESRD and long-term dialysis [5] [6] [7] [8] [16]. Unexpected surgery, surgery without adequate medical optimization, and poor medical condition at the time of surgery are all causes of higher morbidity and mortality in uremic patients who need non-routine orthopedic surgery. A similar situation is seen in geriatric hip fractures. Hip fractures in the geriatric population have become common and result in significant morbidity, mortality, and medical costs [12] [13] [14] [15] [20]. The current study compares and perioperative and postoperative results between these two populations with similar incidence of hip fracture.

The statistical analysis in the current study showed that patients in the uremic group were significantly younger than those in the control group. For our cohort, the number of days between arriving at the ED and having surgery was not significantly higher in the uremic group than in the control group. Each patient underwent a routine cardiac survey before surgery. During the routine cardiac echocardiography examination, every uremia patient was noted to have moderate to severe cardiac pathology including ventricular wall thickening, arrhythmias, valve disorders, and pulmonary hypertension. The presence of underlying cardiac pathology is an important consideration when counseling patients, rela- 
tives and caregivers about the risks of anesthesia and surgery.

Of the 13 complications noted in the uremic patient group, only two were directly related to the hip fracture surgery, and both occurred in two patients with femoral neck fractures. Osteosynthesis surgery for femoral neck fracture in uremic patients undergoing maintenance hemodialysis remains controversial. Fixation with metallic devices has the advantage of being less invasive, with a shorter of operation time, while prosthesis replacement surgery allows weightbearing ambulation immediately. Evidence shows that femoral neck fractures in uremic patients that are treated with osteosynthesis surgery, utilizing either percutaneous screws or hip compression plate fixation, have poor functional results and a high rate of complications including infection, nonunion, and osteonecrosis [21]. In our study, there were 11 patients with femoral neck fractures, and 3 received percutaneous screws fixations. Two of the three fixations performed failed, requiring revised hip prosthesis replacement surgery. The failure rate of osteosynthesis surgery was $66.7 \%$. Only one complication (pneumonia and sepsis) occurred in the prosthesis replacement group of eight patients, although this complication led to the patient's death within the 12-month follow-up period. The death was not directly related to prosthesis failure. Our high failure rate following osteosynthesis surgery for femoral neck fractures in uremic patients is comparable to that reported in the literature [10] [11] [21] [22]. In light of this finding, prosthesis replacement surgery may be the optimal choice of operation for femoral neck fractures in uremic patients, including valgus-impaction fractures and non-displaced fractures.

In comparison there is relative consensus regarding the treatment for intertrochanteric fracture of the femur in uremic patients [10] [11]. As femoral intertrochanteric fracture is considered an extracapsular fracture of the proximal femur, fracture healing is more reliable than that in case of femoral neck fracture after osteosynthesis surgery. In our study, 10 uremic patients with intertrochanteric fracture underwent hip compression screw fixation. Four complications were noted (three patients with arteriovenous fistula failure and one patient with acute heart failure) within 30 days postoperatively; however, no complication was directly associated with osteosynthesis surgery. All fractures were united at a mean time of 11 months yielding a fracture union rate of $100 \%$. We therefore recommend osteosynthesis surgery for repair of femoral intertrochanteric fracture in uremic patients given this high rate success observed after appropriate medical optimization.

There were two surgery-related complications (implant failure) in the uremic group (9.5\%) and eight complications (six patients with implant failure and two with surgical site infection) in the control group (2.6\%). Statistical analysis revealed no significant difference in the rate of surgery-related complications. Additionally, the incidence of surgery-related complications for both groups in the current study was similar to that reported in the literature [10] [23]. The current study also found that the postoperative complication rate was significantly high- 
er in the uremic patients with hip fractures than in the geriatric population. We concluded that the major complications for both the uremic patients and geriatric patients after hip surgery are a result of their medical comorbidities. To our knowledge, this report is the first study to compare postoperative and perioperative results between these two patient groups.

Two uremic patients experienced arteriovenous (A-V) fistula occlusion during their first postoperative hemodialysis treatment. The A-V fistula is the route through which hemodialysis is performed. This treatment is crucial in uremic patients for removing toxins, balancing acid-base status, and normalizing electrolytes, especially after anesthesia and surgical procedures. A failed first renal replacement therapy in these patients may complicate the postoperative situation, and lead to further morbidity and mortality. An emergency embolectomy or thrombectomy was performed for both occluded fistulas, and the patients underwent hemodialysis on the same day. Fortunately, there were no further complications.

We made efforts to limit any bias between the two groups. However, this study has limitations. First, the current study was a retrospective study. Normally, the surgeons and anesthesiologists treat patients conservatively if the risks of surgery and anesthesia are too high. In fact, four patients in the uremic group were treated conservatively, and three died due to their compromised cardiopulmonary condition during admission. This selection bias may have affected the outcomes of our analysis. Second, the analysis included all the selected patients without further stratification. Further studies should distinguish the osteosynthesis group from the prosthesis replacement group. Finally, there were several orthopedic surgeons performing the operations. Although all the surgeons were trained and all worked in the same institute, there were some differences in their surgical technique, approach, and preference regarding the use of implant or prosthesis.

\section{Conclusion}

Although uremic patients are often younger than patients with osteoporosis related hip fractures, anesthesia and surgery carry increased risk for them. Preoperatively, medical optimization is required, and postoperatively, aggressive care should be provided for all complications. For femoral neck fracture in uremic patients, prosthesis replacement surgery may lessen the rate of implant failure, while osteosynthesis surgery for intertrochanteric fractures in the same population has a high fracture union rate. Despite thorough preoperative medical optimization, careful evaluation of operative indication, and intensive postoperative medical care, higher rates of complications are likely to occur due to uremic patients' medical comorbidities.

\section{Acknowledgements}

The authors would like to Miss Shu-Hua Huang for data collection and per- 
forming statistical analysis.

\section{Conflict of Interests}

Yi-Hsun Yu, Yung-Heng Hsu, Ying-Chao Chou, I-Chuan Tseng, Wen-Lin Yeh, Chi-Chung $\mathrm{Wu}$ declare that they have no conflict of interest.

\section{Compliance with Ethical Requirements}

For this type of study, formal consent is not required.

\section{References}

[1] Coco, M. and Rush, H. (2000) Increased Incidence of Hip Fractures in Dialysis Patients with Low Serum Parathyroid Hormone. American Journal of Kidney Diseas es, 36, 1115-1121. https://doi.org/10.1053/ajkd.2000.19812

[2] Ball, A.M., Gillen, D.L., Sherrard, D., Weiss, N.S., Emerson, S.S., Seliger, S.L., Kestenbaum, B.R. and Stehman-Breen, C. (2002) Risk of Hip Fracture among Dialysis and Renal Transplant Recipients. JAMA, 288, 3014-3018.

https://doi.org/10.1001/jama.288.23.3014

[3] Chen, Y.J., Kung, P.T., Wang, Y.H., Huang, C.C., Hsu, S.C., Tsai, W.C. and Hsu, H.C. (2014) Greater Risk of Hip Fracture in Hemodialysis than in Peritoneal Dialysis. Osteoporosis International, 25, 1513-1518. https://doi.org/10.1007/s00198-014-2632-6

[4] Picton, M.L., Moore, P.R., Mawer, E.B., Houghton, D., Freemont, A.J., Hutchison, A.J., Gokal, R. and Hoyland, J.A. (2000) Down-Regulation of Human Osteoblast PTH/PTHrP Receptor mRNA in End-Stage Renal Failure. Kidney International, 58, 1440-1449. https://doi.org/10.1046/j.1523-1755.2000.00306.x

[5] Alem, A.M., Sherrard, D.J., Gillen, D.L., Weiss, N.S., Beresford, S.A., Heckbert, S.R., Wong, C. and Stehman-Breen, C. (2000) Increased Risk of Hip Fracture among Patients with End-Stage Renal Disease. Kidney International, 58, 396-399. https://doi.org/10.1046/j.1523-1755.2000.00178.x

[6] Arneson, T.J., Li, S., Liu, J., Kilpatrick, R.D., Newsome, B.B. and St Peter, W.L. (2013) Trends in Hip Fracture Rates in US Hemodialysis Patients, 1993-2010. American Journal of Kidney Diseases, 62, 747-754. https://doi.org/10.1053/j.ajkd.2013.02.368

[7] Nickolas, T.L., McMahon, D.J. and Shane, E. (2006) Relationship between Moderate to Severe Kidney Disease and Hip Fracture in the United States. Journal of the American Society of Nephrology, 17, 3223-3232. https://doi.org/10.1681/ASN.2005111194

[8] Chang, N.T., Lee, Y.H., Hsu, J.C., Chan, C.L., Huang, G.S., Renn, J.H. and Yang, N.P. (2013) Epidemiological Study of Orthopedic Injuries in Hemodialysis Patients in Taiwan: A Fixed Cohort Survey, 2004-2008. Clinical Interventions in Aging, 8, 301-308. https://doi.org/10.2147/CIA.S41132

[9] Tentori, F., McCullough, K., Kilpatrick, R.D., Bradbury, B.D., Robinson, B.M., Kerr, P.G. and Pisoni, R.L. (2014) High Rates of Death and Hospitalization Follow Bone Fracture among Hemodialysis Patients. Kidney International, 85, 166-173. https://doi.org/10.1038/ki.2013.279

[10] Karaeminogullari, O., Demirors, H., Sahin, O., Ozalay, M., Ozdemir, N. and Tandogan, R.N. (2007) Analysis of Outcomes for Surgically Treated Hip Fractures in Patients Undergoing Chronic Hemodialysis. Journal of Bone and Joint Surgery, 89, 
324-331.

[11] Tosun, B., Atmaca, H. and Gok, U. (2010) Operative Treatment of Hip Fractures in Patients Receiving Hemodialysis. Musculoskeletal Surgery, 94, 71-75.

https://doi.org/10.1007/s12306-010-0080-9

[12] Vaughn, J., Cohen, E., Vopat, B.G., Kane, P., Abbood, E. and Born, C. (2015) Complications of Short versus Long Cephalomedullary Nail for Intertrochanteric Femur Fractures, Minimum 1-Year Follow-Up. European Journal of Orthopaedic Surgery \& Traumatology, 25, 665-670. https://doi.org/10.1007/s00590-014-1557-2

[13] Ha, Y.C., Baek, J.H., Ko, Y.B., Park, S.M. and Song, S.H. (2015) High Mortality and Poor Morbidity after Hip Fractures in Patients with Previous Vertebral Fractures. Journal of Bone and Mineral Metabolism, 33, 547-552. https://doi.org/10.1007/s00774-014-0616-4

[14] Carpintero, P., Caeiro, J.R., Carpintero, R., Morales, A., Silva, S. and Mesa, M. (2014) Complications of Hip Fractures: A Review. World Journal of Orthopedics, 5 , 402-411. https://doi.org/10.5312/wjo.v5.i4.402

[15] Liu, Y., Peng, M., Lin, L., Liu, X., Qin, Y. and Hou, X. (2015) Relationship between American Society of Anesthesiologists (ASA) Grade and 1-Year Mortality in Nonagenarians under Hip Fracture Surgery. Osteoporosis International, 26, 1029-1033. https://doi.org/10.1007/s00198-014-2931-y

[16] Lin, Z.Z., Wan, J.J., Chung, C.R., Huang, P.C., Su, B.A., Cheng, K.C., Chio, C.C. and Chien, C.C. (2014) Epidemiology and Mortality of Hip Fracture among Patients on Hemodialysis: Taiwan National Cohort Study. Bone, 64, 235-239. https://doi.org/10.1016/j.bone.2014.04.017

[17] US Renal Data System (2005) USRDS 2005 Annual Data Report. The National Institute of Health, National Institute of Diabetes and Digestive and Kidney Diseases, Bethesda.

[18] Yang, W.C. and Hwang, S.J. (2008) Taiwan Society of Nephrology. Incidence, Prevalence and Mortality Trends of Dialysis End-Stage Renal Disease in Taiwan from 1990 to 2001: The Impact of National Health Insurance. Nephrology Dialysis Transplantation, 23, 3977-3982. https://doi.org/10.1093/ndt/gfn406

[19] Yu, Y.H., Chen, W.J., Chen, L.H., Niu, C.C., Fu, T.S. and Lai, P.L. (2011) Posterior Instrumented Lumbar Spinal Surgery in Uremic Patients under Maintenance Hemodialysis. Spine, 36, 660-666. https://doi.org/10.1097/BRS.0b013e3181dc9a33

[20] Konstantinidis, L., Papaioannou, C., Blanke, P., Hirschmüller, A., Südkamp, N.P. and Helwig, P. (2013) Failure after Osteosynthesis of Trochanteric Fractures. Where Is the Limit of Osteoporosis? Osteoporosis International, 24, 2701-2706. https://doi.org/10.1007/s00198-013-2392-8

[21] Röden, M., Schön, M. and Fredin, H. (2003) Treatment of Displaced Femoral Neck Fractures: A Randomized Minimum 5-Year Follow Up Study of Screws and Bipolar Hemiprostheses in 100 Patients. Acta Orthopaedica Scandinavica, 74, 42-44. https://doi.org/10.1080/00016470310013635

[22] Haidukewych, G.J., Rothwell, W.S., Jacofsky, D.J., Torchia, M.E. and Berry, D.J. (2004) Operative Treatment of Femoral Neck Fractures in Patients between the Ages of Fifteen and Fifty Years. The Journal of Bone and Joint Surgery. American Volume, 86, 1711-1716. https://doi.org/10.2106/00004623-200408000-00015

[23] Lorich, D.G., Geller, D.S. and Nielson, J.H. (2004) Osteoporotic Pertrochanteric Hip Fractures: Management and Current Controversies. Instructional Course Lectures, 53, 441-454. https://doi.org/10.2106/00004623-200402000-00028 